\title{
A Time-Dose Model to Quantify the Antioxidant Responses of the Oxidative Hemolysis Inhibition Assay (OxHLIA) and Its Extension to Evaluate Other Hemolytic Effectors
}

\author{
M. A. Prieto and J. A. Vázquez \\ Group of Recycling and Valorization of Waste Materials (REVAL), Research Marine Institute (IIM-CSIC), Eduardo Cabello 6, \\ 36208 Vigo, Spain \\ Correspondence should be addressed to M. A. Prieto; michaelumangelum@gmail.com
}

Received 28 February 2014; Revised 16 May 2014; Accepted 28 May 2014; Published 27 August 2014

Academic Editor: Stefano Curcio

Copyright (C) 2014 M. A. Prieto and J. A. Vázquez. This is an open access article distributed under the Creative Commons Attribution License, which permits unrestricted use, distribution, and reproduction in any medium, provided the original work is properly cited.

\begin{abstract}
The development of a convenient mathematical application for testing the antioxidant potential of standard and novel therapeutic agents is essential for the research community to perform evaluations in a more precise form. The in vitro oxidative hemolysis inhibition assay, despite its relevance for in vivo responses, lacks a proper mathematical model to quantify the responses. In this work, a simple nonlinear time-dose tool to test the effectiveness of antioxidant compounds is presented. The model was verified with available experimental data from the bibliography. The model helps to describe accurately the antioxidant response as a function of time and dose allowing comparisons between compounds. Its advantages are a simple application, provision of parametric estimates that characterize the response, simplification of the protocol, economization of experimental effort, and facilitation of rigorous comparisons among the effects of different compounds and experimental approaches. Finally, other effectors that may obstruct or be of interest for the antioxidant determination are also modeled in similar principles. Thus, the basis of more complex multivariable models is provided. In all experimental data fitted, the calculated parameters were always statistically significant, the equations prove to be consistent, and the correlation coefficient of determination was in all cases higher than 0.98 .
\end{abstract}

\section{Introduction}

Antioxidants (A) and prooxidants are chemical entities that can delay or accelerate oxidation processes. Living organisms have developed a complex network $[1,2]$ of enzymatic and nonenzymatic $\mathrm{A}$, which are essential to counteract various harmful prooxidants or reactive species (i.e., $\mathrm{O}_{2}, \mathrm{H}_{2} \mathrm{O}_{2}$, $\mathrm{ROO}^{\circ}$, and $\mathrm{OH}^{\circ}$ ) [3, 4]. Apart from these endogenous A, exogenous ones can derive from natural sources (vitamins, flavonoids, anthocyanins, and some mineral compounds). Clinical trials and epidemiological studies have established an inverse correlation between the intake of natural exogenous A and the occurrence of oxidative stress diseases such as inflammation, cardiovascular problems, cancer, and agingrelated disorders [5-7]. Thus, the analysis of natural antioxidants for disease prevention and the identification of possible prooxidant substances have become topics of growing interest [8-10].

Several in vivo and in vitro methods have been developed for determining the A properties of compounds in order to rank their affinity. In general, these assays differ in their mechanism to generate different radical species and/or target molecules and in the way end-products are measured. At present, there is no convenient assay that enables the evaluation of the antioxidant capacity (AC) for different compounds $[5,11,12]$. The current methods to test the AC still have left many open questions $[13,14]$. The in vitro assays can only rank AC for their particular reaction system and their relevance to in vivo activities is uncertain. Thus, it is logical that, in the last decade, researchers have claimed more cell based approaches [5, 15-17]. Additionally, the arbitrary use of simple analytical procedures to calculate molecular properties, occasionally 
without a validation study, as well as a lack of statistical significance, has caused much controversy [18-23].

OxHLIA is a bioassay for evaluating the inhibition capabilities of A [24], half way between in vivo and in vitro methods. Sheep erythrocytes are subjected to hemolysis by the action of hydrophilic and lipophilic radicals in aqueous system. Hydrophilic radicals are generated from the thermal decomposition of AAPH (2,20-azobis(2-methylpropionamidine) dihydrochloride) attacking the erythrocytes membranes. Lipophilic radicals are derived from the oxidation of the erythrocytes membranes (lipid peroxidation), a phenomena that is initiated by the action of AAPH and thermal induction. The lipophilic and hydrophilic radicals eventually cause the hemolysis of the cell. The time at which the hemolysis occurs depends on the resistance of the erythrocytes population. This hemolytic time can be retarded by antioxidants, capturing the hydrophilic and/or lipophilic radicals. The advantages of OxHLIA are that radicals and substrate targets are biologically relevant compared to other in vitro methods and that antioxidants are subjected to oxidants with different degrees of polarity [25].

Originally, the OxHLIA was performed in a test-tube format, and the degree of hemolysis was spectrometrically determined in the supernatant after centrifugation [24]. Those steps impeded researchers to test large numbers of samples at the same time. Since the development of the method, several studies have improved and extended the applicability of the method [26-29]. Recently, authors [30] have performed a further key modification, which allows following the degree of hemolysis via turbidity of the erythrocyte suspension without centrifugation. This improvement enhances the applicability of OxHLIA into a microplate format. The method thus performed enables evaluation of large numbers of samples of small quantity at the same time with satisfactory precision and reproducibility and in an equivalent way to the previous format procedures.

Its main weakness is the insufficient formalism due to the lack of formal model to describe the kinetic erythrocyte hemolysis, which prevents the quantification of its statistical reliability and loses a part of the relevant information that can be drawn from the experimental results. Therefore, the quantification relies on graphical, or similar methodologies, which causes low reproducibility of the results and leads to an accumulation of procedural restrictions that overstandardize the protocol [31]. Although the meticulous results can be found concerning the kinetics and the factors affecting the reproducibility of the method, the quantification of the results has been left as it was postulated originally [24].

In the current study, a nonlinear mathematical application for describing the OxHLIA responses is developed. It helps to describe accurately the response as a function of time and dose, to provide parameters that summarize the responses, and to facilitate convenient comparisons of the $\mathrm{AC}$ of different compounds. In addition, by analyzing the data of other authors that have studied the effects of other variables or agents over the kinetic erythrocyte population curve, more complex analysis for AC of antioxidants is suggested. Therefore, the basis for postulating more complex multivariable models is also provided. Because the available methodological studies of the OxHLIA appear to have reviewed and extended its procedure into a robust methodology, no improvements of the protocol have been performed.

\section{Material and Methods}

2.1. The OxHLIA Procedure. As recommended by Takebayashi et al. [32], erythrocytes need to be obtained from several adult sheep to reduce the influence of individual differences. Although the common procedure uses sheep erythrocytes, other sources such as human or bovine ones have been proved to produce similar results. Erythrocytes should be washed at least three times with PBS and resuspended in $\mathrm{PBS}$ at a $2.8 \%(\mathrm{v} / \mathrm{v})$ suspension.

2.1.1. Test-Tube Format. As described [32], sheep erythrocytes are suspended at a final concentration in which the maximum hemoglobin liberated cannot saturate the spectrophotometer measuring. The solution is prepared in $0.7 \%(\mathrm{v} / \mathrm{v})$ phosphatebuffered saline (PBS, $\mathrm{pH} 7.4$ ) with $\sim 40 \mathrm{mM}$ of AAPH in the absence (control) and presence of increasing concentrations of antioxidant sample in a test tube at $37^{\circ} \mathrm{C}$ in a water bath with shaking. An aliquot of this mixture was periodically withdrawn every $15 \mathrm{~min}$ for $3 \mathrm{~h}$, and the degree of hemolysis (\%) was determined from the concentration of hemoglobin in the supernatant after centrifugation by measuring the absorbance at $524 \mathrm{~nm}$. The results are expressed as delayed time of hemolysis $(\Delta t)$ as follows:

$$
\Delta t=\tau_{S}-\tau_{C}
$$

where $\tau$ is the time ( $\mathrm{min}$ ) needed to reach the $50 \%$ of the erythrocyte population that is lysed. The subscripts $S$ and $C$ stand for the antioxidant sample and the control, respectively.

2.1.2. 96-Well Plate Format. As described by Takebayashi's et al. work [30], the erythrocyte suspensions $(50 \mu \mathrm{L})$, in the absence or presence of an antioxidant sample $(100 \mu \mathrm{L})$ in PBS, were added to a flat bottom 96-well plate. Complete hemolysis was obtained by adding water to the erythrocyte suspension without sample. The plate was preincubated with a lid at $37^{\circ} \mathrm{C}$; then, AAPH ( $50 \mu \mathrm{L}, 160 \mathrm{mM}$ in PBS) was added to initiate the assay and incubated at $37^{\circ} \mathrm{C}$ in the incubator with shaking. The optical density at $660 \mathrm{~nm}$ was measured every $10 \mathrm{~min}$. The percentage of survival erythrocyte population $(P)$ was calculated as follows:

$$
P=\left(\frac{n_{t}-n_{\max }}{n_{0}-n_{\max }}\right) \times 100,
$$

where $n$ is the optical density measure at the start of the reaction ( 0$)$ or at any $t(\mathrm{~min})$ and $n_{\max }$ is the maximum optical density of the complete hemolysis. Then, the time to reach $50 \%$ of the survival population $(\tau)$ is obtained graphically for an increasing concentration of an antioxidant. Afterwards, (2) is used to normalize the $\tau$ values formulated by (1). 
2.1.3. Quantification. The kinetic part is solved in general by a graphical method. In the test-tube and microplate approaches, the $\Delta t$ values obtained are analyzed in liner terms as a function of the antioxidant concentration. The slope $([\mathrm{A}] / \mathrm{min})$ of such analysis is used as the comparative value between different compounds. Although such a double approach is correct, it seems contradictory that the algebraic tools are reserved only to the second part of the procedure, which prevents the statistical validation of the results as a whole. The statistical information of the parameters found in the first graphical step is lost, and even if we compute the parametric estimations of the second step, it does not take into account the variable involved in the first step. Thus, among other problems, the two-step procedure lacks a proper statistical estimation.

2.2. Data for Model Verification. The bibliographical abundance about antioxidant activity in a competitive reaction, in raw and purified extracts, makes it practically superfluous to extend the experimental work, specifically devoted to validate the model proposed here. In this respect, its descriptive accuracy was verified using results from other authors (taken from the published figures by means of GetData Graph Digitizer 2.24), selected in such a way that they implied different methods, substrates, and time domains. Table 1 shows a detailed description of the references used, the corresponding figures subjected to analysis, the variable or agent involved, and some relevant conditions of the assays. As it will be noted, the studies from which the data was collected are those that had been involved in developing, reviewing, or extending the methodology responses evaluated here. We hope that the reader would find them as key data, rather than a lack of experimental effort.

2.3. Numerical and Statistical Methods. Fitting the experimental results to the proposed equations was carried out in two phases. First, parametric estimates were obtained by minimization of the sum of quadratic differences between observed and model-predicted values, using the nonlinear least-square (quasi-Newton) method provided by the macro Solver in Microsoft Excel 2003, which allows quick testing of hypotheses and the display of their consequences. Next, the determination of the parametric confidence intervals and model consistency (Student's $t$-test and Fisher's $F$-test, respectively, in both cases with $\alpha=0.05$ ) was calculated using the "SolverAid" [33]. The "SolverStat" macro [34, 35] was used for detecting possible anomalies in the distribution of parametric estimates and residuals.

\section{Results and Discussion}

In general, the bioassay studied here is of a special elegance and applicability. One of the major drawbacks is the lack of formal model to describe the kinetic erythrocyte hemolysis. Therefore, the quantification of the hemolytic time $\tau$ (time to reach the $50 \%$ of the survival population of erythrocytes) has to be obtained graphically or similarly from the kinetic erythrocyte survival curve and thus, the results provided lack of relevant statistical information. Additionally, such a lack of mathematical expression causes low reproducibility of results, which often leads to accumulation of procedural restrictions that could overstandardize the protocol. Despite the existence of very rigorous results about the kinetics and the factors affecting the reproducibility of the method, for some reasons, the quantification of the results has been left as it was postulated originally [24].

Next, we will briefly review different mathematical methodologies from related fields of study, such as the antioxidant, dose-response, and hemolytic bioassays. Afterwards, based on such analysis, a simple illustrative bivariate (simultaneous analysis of time and effector responses) mathematical application for many different effector agents that affect the hemolytic process is presented. Then, a highly appropriated alternative model for OxHLIA is verified by using data from other authors that had investigated the capacity of several antioxidants in the OxHLIA reactions. Finally, other effectors that may obstruct or be of interest for antioxidant determination are modeled in similar principles as those defined in the OxHLIA reaction.

3.1. Modeling Approaches from Related Fields of Study. Besides other authors [41-43], we reject the simplistic ways of characterization of antioxidant action and we attempt to address this issue by bringing across well-established ideas from existing fields to overcome the existing problems for the quantification OxHLIA antioxidant bioassay. Table 2 shows a short review of different mathematical expressions from related fields of study that provide the key knowledge to guide the development of appropriate solution for the OxHLIA antioxidant bioassay. At present, the reaction mechanism of the erythrocyte lysis is well understood. Authors have developed a sophisticated mechanistic model to evaluate the erythrocyte lysis [39]. The detailed mechanistic description of oxidation is complex because the heterogeneity of the cell population varies from one to the other systems making it difficult to characterize the responses and measure all the compounds involved in the reaction. Therefore, researches tend to search for empirical general models [28, 40], able to quantify and evaluate the complex phenomenon of hemolysis. In this regard, authors [38] have demonstrated the suitability of the Weibull survival distribution connecting its parameters to blood properties.

From the antioxidant field, due to the complexity of the oxidative reactions, multiple number of simple calculation procedures and different mechanistic or empiric kinetic models $[41-43,48]$ are routinely used to compute the inhibitory responses of different agents. Among the available nonlinear models, the power function developed by Terpinc and Abramovič [42] is only appropriate to adjust fractional-order kinetic profiles, but it fails in the description of first-order processes or sigmoidal profiles. The Logistic and Weibull equations, which have been transferred from microbiology and pharmacology dose-response fields to describe the oxidation action [41, 43], are more appropriate equations for modeling oxidation processes. 


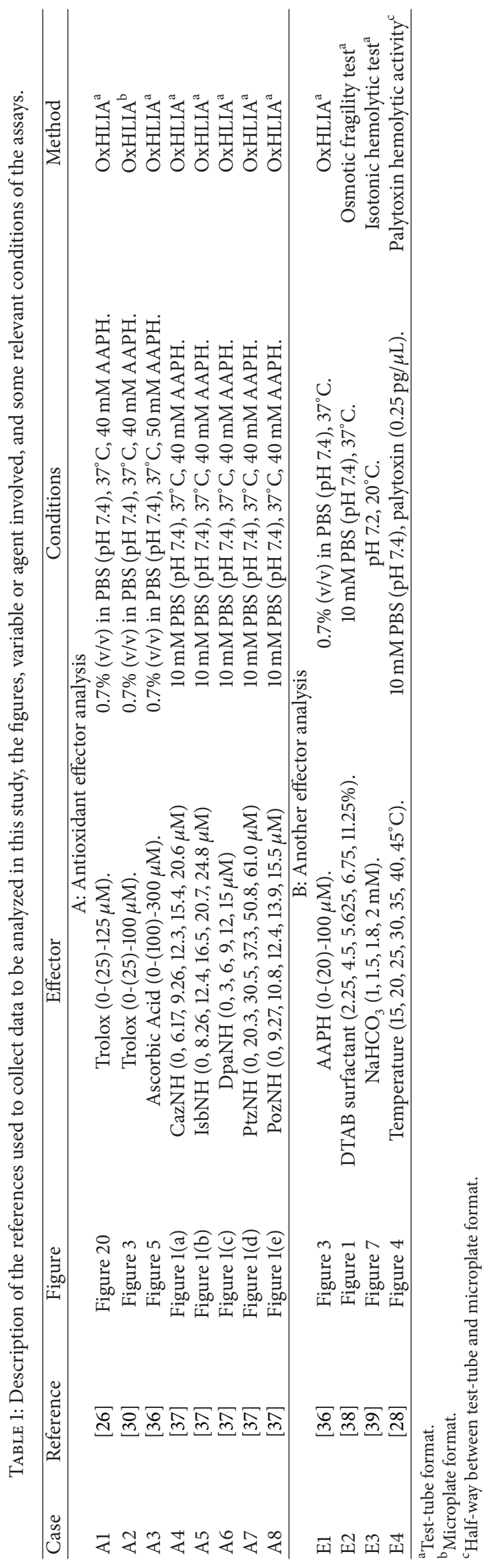




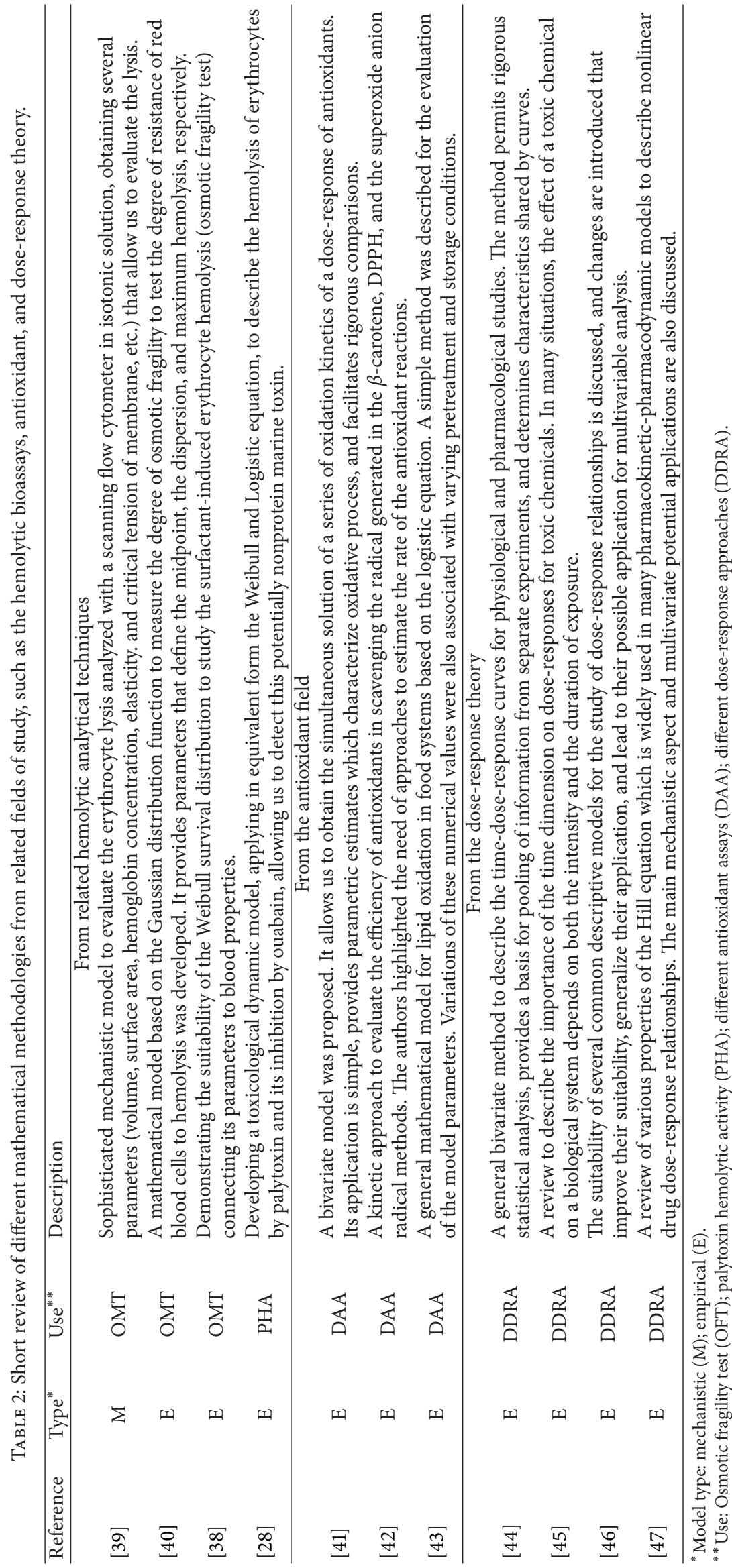


From the dose-response theory, the three-parameter sigmoidal group of functions (such as the Logistic, Weibull, Hill, Gompertz or Richards-Chapman) would be, in general, acceptable solutions to fit individually the kinetic profiles corresponding to a series of increasing level agents or variables[44-47].

In general, most of the above models can be transferred to study the erythrocyte survival analysis found in the OxHLIA method and help to compute, in a proper form, the inhibitory features over the control curve produced by any antioxidant agent. Those models would be able to produce key parameters to summarize the responses, such as the asymptote, half-life, maximum velocity, or the lag-phase, and they can be used to quantify the effect of different chemicals. However, in the following, by merging all the above solutions, we will describe in detail what we consider as suitable solution for the OxHLIA antioxidant bioassay.

\subsection{Mathematical Modeling for the Kinetic Description of the Survival Erythrocyte Population}

3.2.1. Normalization. In an open system, in the presence of enough quantity of initiator (AAPH) and in the absence of an antioxidant, the peroxidation of the erythrocyte membrane would be oxidized exhaustively at sufficiently long times, causing its rupture, producing asymptotic responses that would correspond to the erythrocyte population mixed in the solution. Consequently, the spectrophotometric responses $(R)$ should be carefully standardized. Therefore, before the data is analyzed, the response $(R)$ must be normalized as a function of the maximum hemolysis $\left(R_{\max }\right)$ that can be achieved. For example, a simple way could be to compute the $R$ in terms of survival population $(P)$ in percentage as follows:

$$
P=\left(\frac{R}{R_{\max }}\right) \times 100 .
$$

Normalization of the raw data through multiplication adjusts the resulting sample variation (human error, excessive or defective dilution, number of red blood cells that are present, and so forth). Fitting the normalized data resulted in a significant increase in the reproducibility and accuracy of the results obtained, but no significant change in the parameters was produced.

3.2.2. Kinetic Description of the Survival Erythrocyte Population (Univariate Approach). The above previous works $[28,38,40]$ reflect as the most appropriate alternative the use of sigmoidal profiles to describe the kinetic hemolysis of erythrocytes. In accordance with other authors, we have also found that the Weibull survival distribution [49] is a suitable solution for the analysis of OxHLIA survival responses. The function can be formulated in terms of the variables $t$ (time) and $P$ (survival erythrocyte population) in its original form as follows:

$$
\frac{P}{100}=\exp \left[-\left(\frac{t}{b}\right)^{a}\right]
$$

where $a$ and $b$ are parameters of form and position, respectively. Its use, in our context, requires two modifications: (1) to multiply the second member by the maximum response $K$, to assure that the asymptote can take values different from $100 \%$ in case any antioxidant or other variable may be able to protect a partial erythrocyte population from hemolysis and (2) to reparametrize the equation in such a way that it would include explicitly the time for the substrate half-life $(\tau)$, which is the desirable parameter in this type of bioassay. Therefore, the time course of the survival population of erythrocytes can be postulated to occur as function of the parameters variation as in the following equation:

$$
P=K \exp \left[-\ln 2\left(\frac{t}{\tau}\right)^{\alpha}\right]
$$

where $K$ is the asymptote, $\tau$ is the time required for $50 \%$ oxidation (substrate half-life), and $\alpha$ is shape parameter related to the maximum slope of the response. Equation (5) is very versatile: when $\alpha<1$, it can adjust the profiles of potential responses; when $\alpha=1$, a first-order kinetic is described; when $\alpha>1$, a variety of sigmoidal profiles are produced.

3.2.3. Additional Parameters of Interest. Apart from the $\tau$ parameter, other interesting ones can be obtained, such as the maximum rate of hemolysis $\left(v_{m}\right)$, the rate at the $\tau$ value $\left(v_{\tau}\right)$, and the lag-phase $(\lambda)$. The rate values can be obtained with some algebraic modifications from (5) as presented in Table 3 (part A). Regarding the appearance of a lag-phase, since no induction period was observed in micelle model systems, this feature of the profile in the OxHLIA survival responses is attributed [50] to the action of antioxidants present in the erythrocyte membrane. The confidence intervals of $v_{m}, v_{\tau}$, and $\lambda$ could be estimated by means of the reparameterization of (5) to make such values explicit (Table 3, part A), but the result is less operative than the robust estimation of the $\tau$ parameter [31].

3.2.4. Application to Bibliographic Antioxidant Data. Figure 1(a) shows the typical time-dose response of hemolysis curves using the antioxidant trolox at various concentrations $0-(25)-125 \mu \mathrm{M}$ (dots). The results were obtained from the study of Takebayashi et al. [26] (Case A1 in Table 1) who recently published a detailed revision of the method. The collected data was normalized by (3) and fitted (lines) to the kinetic survival function (5). Parametric estimations are shown in Table 4. Statistically significant results were found continuously (Student's $t$-test, $\alpha=0.05$ ), the equation was consistent (Fisher's F-test), and the goodness of fit coefficient of determination was higher than 0.98. Figures 1(b), 1(c), and $1(\mathrm{~d})$ show the pattern of the parametric responses as a function of the antioxidant concentration. The parameters $\tau$ and $\lambda$ show a linear behavior, while the rate parameters of $v_{\tau}$ and $v_{m}$ display a decreasing asymptotic hyperbolic relation. Any of those parameters can be easily described via simple functions. Such a description produces detailed information, summarized in parameters that can be used to compare the AC of compounds, as an example.

Most of this information is already well-known [24, 30, 41]. However, authors $[30,32,36]$ obtain the $\tau$ parameter, the 
TABLE 3: Part A shows the additional parameters of interest $\left(v_{m}, v_{\tau}\right.$, and $\left.\lambda\right)$ and reparameterization equations deduced from algebraic modifications of (5) to make such values explicit and therefore to compute their confidence intervals. Part B shows four of the most common effector perturbations (linear, hyperbolic, sigmoidal, and bell modifications) on the kinetic description of the survival erythrocyte population.

\begin{tabular}{|c|c|c|}
\hline \multicolumn{3}{|c|}{ A: Additional parameters of interest } \\
\hline Parameter calculation & Reparameterization form of (5) & Eq. number \\
\hline$v_{m}=\frac{K \alpha \ln 2}{\tau} G^{G} e^{-G}$ & $P=K \exp \left[-(\ln 2)^{1-\alpha}\left(\frac{e^{G} v_{m} t}{K \alpha G^{G}}\right)^{\alpha}\right]$ & (i) \\
\hline$v_{\tau}=\frac{K \alpha \ln 2}{2 \tau}$ & $P=K \exp \left[-(\ln 2)^{1-\alpha}\left(\frac{2 v_{\tau} t}{K \alpha}\right)^{\alpha}\right]$ & (ii) \\
\hline$\lambda=\frac{\tau}{\sqrt[\alpha]{\ln 2}}\left[G^{1 / \alpha}+\frac{e^{-G}-1}{\alpha G^{G} e^{-G}}\right]$ & $P=K \exp \left[-\left(\left(G^{1 / \alpha}+\frac{e^{-G}-1}{\alpha G^{G} e^{-G}}\right) \frac{t}{\lambda}\right)^{\alpha}\right]$ & (iii) \\
\hline \multicolumn{3}{|c|}{ B: effector variations on the survival population } \\
\hline Effector relation & Model chosen to modify parameters of (5) & Eq. no. \\
\hline Linear $(L)$ & $L(e)=e \cdot l_{1}+l_{2}$ & (iv) \\
\hline Hyperbolic $(H)$ & $H(e)=h_{1}\left[1-\exp \left(h_{2} \cdot e\right)\right]$ & (v) \\
\hline Sigmoidal $(S)$ & $S(e)=s_{1} \exp \left[-\ln 2\left(e / s_{2}\right)^{s_{3}}\right]$ & (vi) \\
\hline Bell $(B)$ & $B(e)=b_{1}\left\{\frac{b_{3}}{b_{2}}\left[1-\left(\frac{e}{b_{4}}\right)^{b_{2}}+\ln \left(\frac{e}{b_{4}}\right) b_{2}\right]\right\}$ & (vii) \\
\hline
\end{tabular}

Part A: maximum rate of hemolysis $\left(v_{m}\right)$; the rate at the value $\left(v_{\tau}\right)$; the lag-phase $(\lambda)$; and $G=(\alpha-1) / \alpha$.

Part B: $l_{1}$ is the slope ( $t / e$ units); $l_{2}$ is the intercept ( $t$ units); $h_{1}$ is the asymptotic value of the hyperbolic relation (parameter modified units); $h_{2}$ is $1 / e$ units; $s_{1}$ is the asymptotic value (parameter modified units) of the nonlinear relation, $s_{2}$ is the $\mathrm{IC}_{50}$ value ( $e$ units); $s_{3}$ is a shape parameter related to the maximum slope of the response; $b_{1}$ is the maximum value (parameter modified units), $b_{2}$ is related to the distance between the tails of the function ( $e$ units), $b_{3}$ is a value related to the asymmetry of the bell profile, and $b_{4}$ is the effector value at which $b_{1}$ takes place ( $e$ units).

TABle 4: Parametric estimations and statistic information of the kinetic series of the survival erythrocyte population inhibited by the antioxidant trolox and fitted to the kinetic model (5).

\begin{tabular}{|c|c|c|c|c|c|c|c|}
\hline \multirow{2}{*}{$\begin{array}{l}\text { Effector } \\
\text { Trolox }(\mu \mathrm{M})\end{array}$} & \multicolumn{3}{|c|}{ Kinetic parameters } & \multirow{2}{*}{$\begin{array}{c}\text { Statistics } \\
\qquad R_{\mathrm{adj}}^{2}\end{array}$} & \multicolumn{3}{|c|}{ Reparameterization } \\
\hline & $\begin{array}{c}K \\
\text { (\% Ert) }\end{array}$ & $\begin{array}{c}\tau \\
(\min )\end{array}$ & $\alpha$ & & $\begin{array}{c}v_{\tau} \\
(\% \mathrm{Ert} / \mathrm{min})\end{array}$ & $\begin{array}{c}v_{\max } \\
(\% \text { Ert } / \min )\end{array}$ & $\begin{array}{c}\lambda \\
(\min )\end{array}$ \\
\hline 0 & $100 \pm 1.15$ & $68.12 \pm 10.03$ & $4.59 \pm 2.48$ & 0.9992 & $2.34 \pm 1.01$ & $2.35 \pm 0.88$ & $39.92 \pm 12.12$ \\
\hline 25 & $100 \pm 1.15$ & $86.05 \pm 10.63$ & $6.44 \pm 5.17$ & 0.9993 & $2.59 \pm 0.98$ & $2.63 \pm 0.47$ & $59.87 \pm 17.22$ \\
\hline 50 & $100 \pm 1.15$ & $102.96 \pm 10.74$ & $8.26 \pm 7.73$ & 0.9984 & $2.78 \pm 0.75$ & $2.85 \pm 0.65$ & $78.16 \pm 21.34$ \\
\hline 75 & $100 \pm 1.15$ & $117.26 \pm 10.53$ & $10.14 \pm 6.89$ & 0.9988 & $3.00 \pm 0.68$ & $3.09 \pm 0.32$ & $94.04 \pm 18.75$ \\
\hline 100 & $100 \pm 1.15$ & $130.76 \pm 10.47$ & $11.20 \pm 6.92$ & 0.9987 & $2.97 \pm 0.59$ & $3.06 \pm 0.89$ & $107.22 \pm 21.24$ \\
\hline 125 & $100 \pm 1.15$ & $144.26 \pm 10.60$ & $10.86 \pm 8.30$ & 0.9971 & $2.61 \pm 0.85$ & $2.69 \pm 0.97$ & $117.51 \pm 31.25$ \\
\hline
\end{tabular}

most consistent one, by graphical analysis, and then a linear analysis is used to analyze the dose effect. Occasionally, (5) or similar are used [37] to extract the relevant information from the kinetic survival curve and then, the dose effect over the $\tau$ parameter is analyzed separately in linear terms. As it has being done in Figure 1, the analysis is performed in a twostep procedure. The statistical information of the parameters found in the first step is lost in the second one. Even if we compute the parametric estimations of the second step, it does not take into account the variable involved in the first step. Thus, among other problems, the two-step procedure lacks a proper statistical estimation.

When the kinetic OxHLIA survival responses were produced in the test-tube format, simple approaches seem to be acceptable. However, since the development of the microplate procedure, effortless time-dose data can be obtained and more complex or robust solutions are recommended. Next, general alternatives, including those to describe the antioxidant responses to build simultaneous solutions for most typical modifications of the kinetic erythrocyte survival curve by common tested effectors, are discussed.

3.3. Mathematical Model of Effectors Altering the Kinetic Description of the Survival Erythrocyte Population. The development of a theoretical model is greatly facilitated by the possibility of combining all experimental data into a single master curve that is able to account for the important 
First fitting step

Univariate kinetic anlaysis with Weibull function

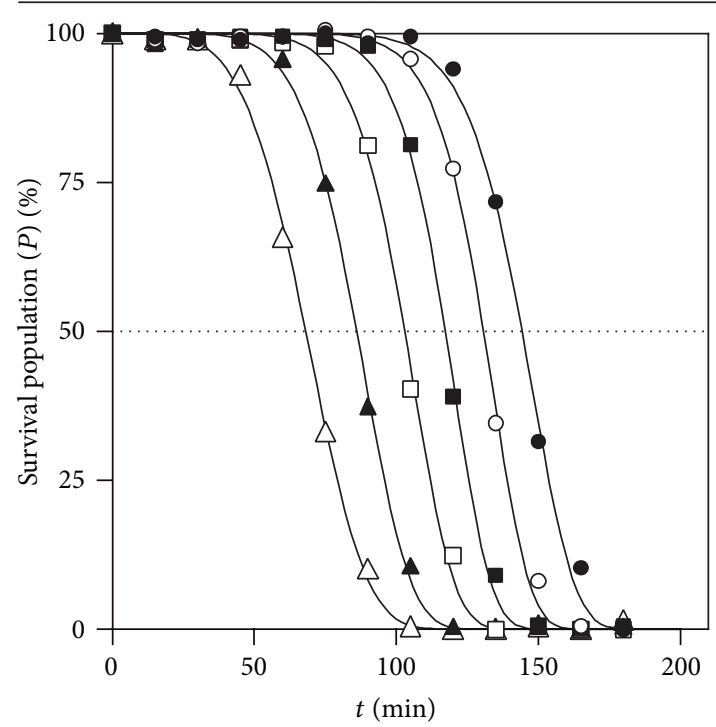

(a)

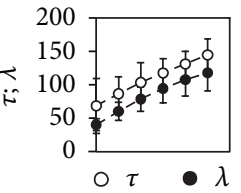

(b)

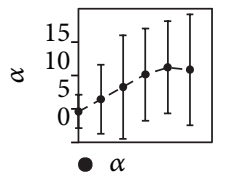

(c)

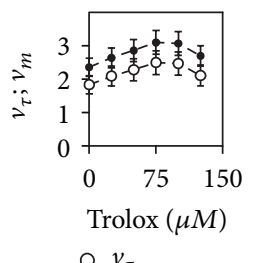

- $v_{m}$
Second fitting step

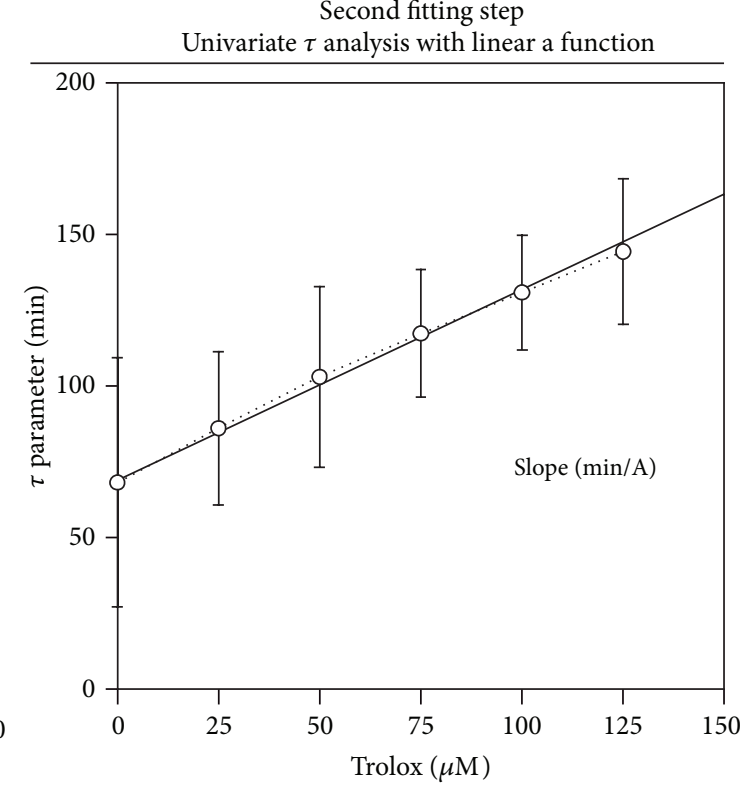

(e)

(d)

Figure 1: Kinetics of the haemolytic oxidation at different antioxidant concentrations and relationships among the concentration of antioxidant and the parameters that characterize its activity. (a) The kinetic series of the survival erythrocyte population fitted (lines) to the kinetic model (5) using the antioxidant trolox at various concentrations 0-(25)-125 $\mu \mathrm{M}$ (dots). The results were obtained from the study of Takebayashi et al. [26] (Case A1 in Table 1) who recently made a detailed revision of the method. Parametric estimations are showed in Table 4. (b), (c), and (d) show the pattern of the parametric responses as a function of the antioxidant concentration. The parameters $\tau$ and $\lambda$ show a linear behavior, while the rate is the modified final $v_{\tau}$ and $v_{m}$ displaying a decreasing asymptotic hyperbolic relation.

variables simultaneously. Such a solutions allows us to control most factors that affect the system, helping to standardize the key variables for producing reproducible protocols and therefore, to obtain reproducible results. Despite the existence of very rigorous results regarding the kinetics and the factors affecting the kinetic curve of the survival erythrocyte population, simultaneous solutions are not always performed, and by applying them, we can provide key knowledge to understand partially the governing mechanisms.

\subsubsection{Possible Effector Variations on the Kinetic Description} of the Survival Erythrocyte Population. We considered an effector as a variable or chemical entity that can cause a shape variation on the kinetic curve of the survival erythrocyte population, for example, the surfactant or $\mathrm{NaCl}$ concentration, typically applied in the osmotic fragility test; environmental variables such as $\mathrm{pH}$ and temperature; prooxidants such as AAPH; antioxidant agents. Unfortunately, due to the diversity and complexity of the perturbations of those effectors over the survival erythrocyte population curve, it is complex to postulate one empiric model that could be applied indiscriminately to produce all corresponding shape variations. The perturbations caused can be very different and affect one or various parameters of (5) (or any other reparametrized form). In general terms, four of the most common perturbations (linear, hyperbolic, sigmoidal, and bell modifications) over the kinetic curve of the erythrocyte survival population are summarized in Table 3 (part B).

Those pattern descriptions suggest different mechanisms of the cell lysis process. However, as in the previous section, because of the heterogeneity of the mechanistic reactions, it is difficult to characterize in mechanistic terms the hemolysis. Therefore, modeling with phenomenological approaches is the most appropriate solution to consider. When the previous phenomenological models are used we are not uncovering the mechanistic relations, but the hemolytic responses are a complex set of sequential and parallel reactions that represent, to an extent, one of the phenomenological curves.

In this regard, linear variations over the parameter $\tau$ have been found when describing the antioxidant inhibitory effects over the erythrocyte survival curve [24, 26, 30]. Hyperbolic ones have been found, when describing the effects of prooxidant agents, such as AAPH [36]. Sigmoidal effects also have been found, when describing the concentration of the surfactant or agents such as $\mathrm{NaCl}$ or the toxic palytoxin $[28,38,40]$. Bell profiles are normally found when analyzing the effects of variables such as temperature or $\mathrm{pH}$ [28].

The reader should note that the equations available to describe $H, S$, and $B$ relations are diverse. We have selected the ones that we felt appropriate, but many different ones could be used. 
3.3.2. Simultaneous Solution to Describe the Effector Modifications over the Kinetic Curve of the Survival Erythrocyte Population: A Bivariate Approach. To introduce any of the previous phenomenological effector functions $(f)$ affecting the survival erythrocyte (5), but without altering the kinetic parameters, such $f$ should multiply any of its parameters $(\theta)$ as follows:

$$
m_{\theta}=\theta_{C} \times[1+f(e)] ; \quad \text { where }(\theta=K, \tau, \alpha),
$$

in which $m_{\theta}$ is the modified final function for the parameters $\theta$ present in (5). Thus, when any parameter $\theta$ is perturbed by $f(e)$, it becomes also a function of the variable $e$, but its original value without perturbation (or control, $\theta_{C}$ ) remains intact, and so does its statistical information. Therefore, a bivariate equation can be formulated, as a function of $t$ and the modifier $e$, increasing significantly the descriptive capabilities of the model for real cases. In its more complete case, the proposal would be as follows:

$$
P(t, e)=m_{K} \exp \left[-\ln 2\left(\frac{t}{m_{\tau}}\right)^{m_{\alpha}}\right] .
$$

In consequence, when an entire set of kinetic profiles is simultaneously described by (7), the term $m_{\theta}$ typifies the specific variation, characterized by the mechanism caused due to the effector variable. Such a characterization allows using one (in the case of $L$ relation) or various parameters (for $H, S$, or $B$ relations) of $m_{\theta}$ and its statistical information for different purposes. This multivariable characterization is especially robust, minimizing the effects of random and systematic errors. As stated by many authors before [44, 51], optimally and efficient data analysis should involve simultaneous description of all curves, rather than fitting each one individually. The simultaneous curve-fitting reduces the number of parameters needed to analyze the response, which is a more informative approach and provides better estimations of parameters, and finally reduces their interval of confidence [31]. In addition, once all the modes of action are mathematically known, if the experimental curves obtained do not span the full range and some of them fail to provide information about one or more of the parameters of the equation, the bivariate application describes simply and accurately all the responses.

The reader should note that we have selected (5) as the principle model to perform the analysis of the effector agent. Thus, we have selected the form that makes explicitly the $\tau$ parameter and avoided the other possible reparameterization forms that provide other parameters of interests $\left(v_{m}, v_{\tau}\right.$, and $\lambda$ ). Such a preference, shared by many other authors in many different fields, should not be a restriction. Those other reparameterization forms could be used, but the essence of the simultaneous approach should be kept.

In the following, the specific OxHLIA survival responses produced by the action of antioxidant effectors are analyzed in detail first, in which a simple simultaneous time-dose model is presented. The model is verified using kinetic data effects of dose response of several antioxidants collected from the bibliography. Secondly, other typical modifications by other effectors are described in bivariate terms, extending the versatility and applicability of simultaneous approaches.
3.4. Verification of the Bivariate Procedure with Experimental Data from Bibliographic Results to Describe Antioxidant Agents as Effectors. An oxidation inhibitor can be characterized in detail by the variations caused in the parameters of (5), all of them with precise meanings, as well as theoretical and practical interest with respect to the oxidation kinetics. In open systems, the asymptotic parametric value $(K)$ will always reach the maximum oxidizable value (in this case the $100 \%$ of the erythrocyte population). Thus, as a function of an A effector, the $K$ parameter should not be modified by its action and should remain constant. Regarding the parameters $\tau$ and $\alpha$, as suggested by the authors [24,30], they should be modified by $L$ relation. Therefore, by inserting relation ((iv) Table 3) modifying the parameters $\tau$ and $\alpha$ of (5), a bivariate model is obtained, as a simultaneous function of time and the modifier concentration. Figure 2 shows the antioxidant (Table 1, Case A1) presented in Figure 1, analyzing simultaneously the time and the dose. The statistical results are displayed in part A of Table 5 (Case A1). From such determination, the parameter $l_{1}(\mathrm{~min} / \mu \mathrm{M}$ of trolox $)$, modifying the $\tau$ parameter of the kinetic curve of the survival erythrocyte population, provides the key relevant information for $\mathrm{AC}$ comparisons.

When we assume that the half-life $(\tau)$ is linearly modified by the presence of an antioxidant, such a behavior translates that each antioxidant molecule is able to capture the same amount during the time of the assay, independently of the type and the degree of polarity of the radicals generated. Also, it implies that in solution the antioxidants stability remains persistent during the time of the assay. This pattern has been found in other kinetic responses from different methodologies [41]. Figure 3 shows the analysis for other five cases collected from literature data (A2-6, Table 1). As in the previous case, the data could be modeled by inserting the linear relation ((iv) Table 3) modifying the parameters $\tau$ and $\alpha$ of (5). Parametric estimations and statistical information of the fittings are found in Table 5. Linear variations are successfully applied to the data published by different authors, and when we simultaneously allowed nonlinear relations, such as $H$ or $S$, occasionally some improvements are obtained (data not showed). However, this saturated effect was weak and most of the attempts showed a nonsignificant solution of at least one parameter. The nonlinear pattern descriptions could uncover a saturated effect over the hemolytic reaction. This saturated response behavior has been already described in similar antioxidant responses by means of a hyperbolic effect over one or several parameters of the equation describing the kinetic oxidation, for example, the responses described in the LDL [52], $\beta$-carotene [53], and crocin [54] assay reactions to evaluate antioxidants. In any case, the apparently consistent linearity of the responses provides a simple solution to quantify the OxHLIA survival responses (Figure 3 and Table 5).

3.5. Extending the Analysis to Other Effectors. It is known that other effectors, such as metals, surfactants, and temperature or light intensity, are harmful to cell membranes, damaging its structure and eventually causing hemolysis. 
TABLE 5: Numeric results corresponding to the time-dose antioxidant responses collected from the bibliography (enumerated in Table 1) adjusted to (7) perturbed by (iv) in Table 3.

\begin{tabular}{lcccccc}
\hline & & & & \multicolumn{2}{c}{ Parametric estimates } & \multicolumn{2}{c}{ Statistics } \\
& Effector & $K$ & Kinetic parameters & \multicolumn{2}{c}{ Effector modifying coefficients } & $R_{\text {adj }}^{2}$ \\
\hline A1 & Trolox & $100.0 \pm 0.5$ & $70.5 \pm 0.9$ & $4.83 \pm 6.9$ & $0.0086 \pm 2.2$ & $0.0121 \pm 16.5$ \\
A2 & Trolox & $100.0 \pm 0.3$ & $84.2 \pm 0.4$ & $5.50 \pm 3.6$ & $0.0063 \pm 1.4$ & $0.0115 \pm 9.7$ \\
A3 & Ascorbic Acid & $100.0 \pm 1.1$ & $139.8 \pm 1.8$ & $4.97 \pm 12.4$ & $0.0032 \pm 4.6$ & $0.0030 \pm 37.2$ \\
A4 & CazNH & $100.0 \pm 1.1$ & $199.7 \pm 1.3$ & $5.03 \pm 12.2$ & $0.0470 \pm 0.3$ & $-0.0092 \pm 11.9$ \\
A5 & IsbNH & $100.0 \pm 1.0$ & $208.1 \pm 1.4$ & $5.07 \pm 7.5$ & $0.0356 \pm 3.9$ & $-0.0073 \pm 46.8$ \\
A6 & DpaNH & $100.0 \pm 1.7$ & $222.6 \pm 1.8$ & $6.48 \pm 11.5$ & $0.0798 \pm 4.4$ & $-0.0169 \pm 47.8$ \\
A7 & PtzNH & $100.0 \pm 0.8$ & $209.0 \pm 1.2$ & $6.65 \pm 7.9$ & $0.0184 \pm 2.9$ & $-0.0031 \pm 47.4$ \\
A8 & PozNH & $100.0 \pm 0.9$ & $186.5 \pm 1.3$ & $9.09 \pm 11.3$ & $0.1014 \pm 2.6$ & -0.02937 \\
\hline
\end{tabular}

Confidence intervals for $\alpha=0.05 . R_{\text {adj }}^{2}$ : adjusted determination coefficient.

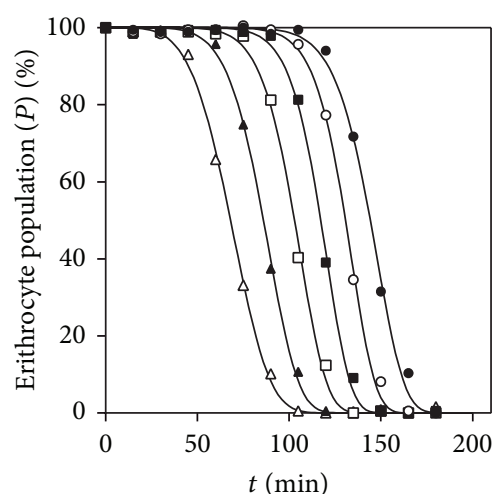

(a)

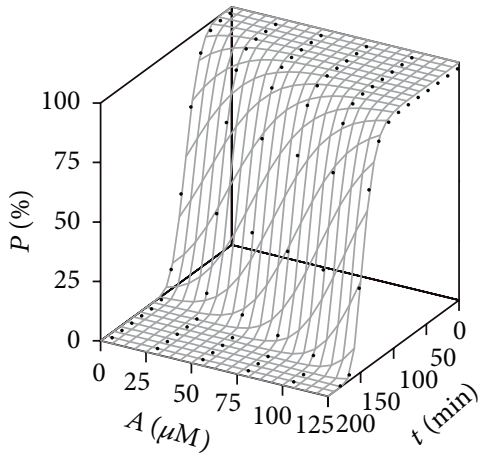

(b)

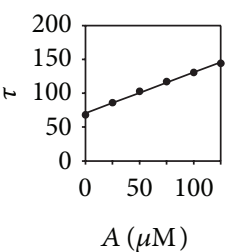

(c)

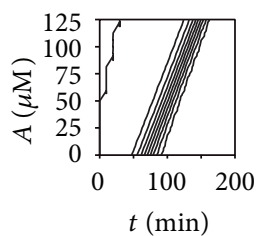

(e)

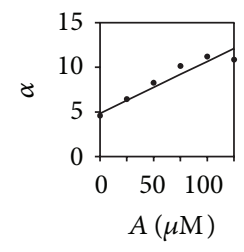

(d)

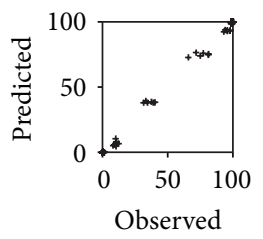

(f)

Figure 2: Trolox antioxidant case (Case A1, Table 1), presented in Figure 1 and analyzed simultaneously in terms of time and the dose by model (7). Statistical results in part A of Table 5 (Case A1).

Such effectors, such as the antioxidant concentration, are dose-time dependent. Those effectors can be the interest in the AC determination since they can also destabilize AC. Understanding and describing its effect over the kinetic erythrocyte population curve are crucial in order to be able to quantify the changes that those effectors may produce.

More specifically, surfactants not only cause hemolysis, but also are commonly used to help hydrosoluble compounds to be solubilized in lipophilic environments or inversely. In this sense, in the OxHLIA analysis, researches could be interested to investigate how a hydrophilic A may be able to penetrate beyond the surface of the erythrocyte membrane, by adding different concentrations of a surfactant while studying the time-dose effect of an A. We have not found any bibliographic data available, but there are a plenty of studies that deal with the hemolytic effect of surfactants. Therefore, by being able to describe its effect in time- and effectordependent form, we are providing the basis for postulating more complex multivariable models (time, antioxidant, and surfactant dose effect). Also in the case of surfactants, other agents or variables may be of interest to determine its effect to provide the base for more intriguing effects over the $\mathrm{AC}$ of compounds in the OxHLIA, such as the temperature or prooxidant agents.

Although the modifications produced by other variables (or effectors) over the kinetic erythrocyte population curve have been studied in detail and in many diverse reactions, this bibliographic data in many occasions is incomplete in the variable range studied or the effect is mixed with other variables. In addition, the data frequently is presented only in a $3 \mathrm{D}$ graph, which makes its data digitization nearly impossible. Thus, after an extensive search, only a small quantity of available examples could be used to test the general applicability of the general master model proposed. Table 1 (part B) shows the examples selected. They involved different effectors of different hemolytic assays, affecting the kinetic survival erythrocyte population curve.

The results of applying kinetic model (5) or any of its other reparameterization forms to the survival erythrocyte population curve affected by different effectors varying one or more parameters with one or various of the phenomenological functions described-Equations ((iv), (v), (vi), and (vii) Table 3) - are presented in Figure 4 independently of the mechanistic interpretation that can be inferred by analyzing 

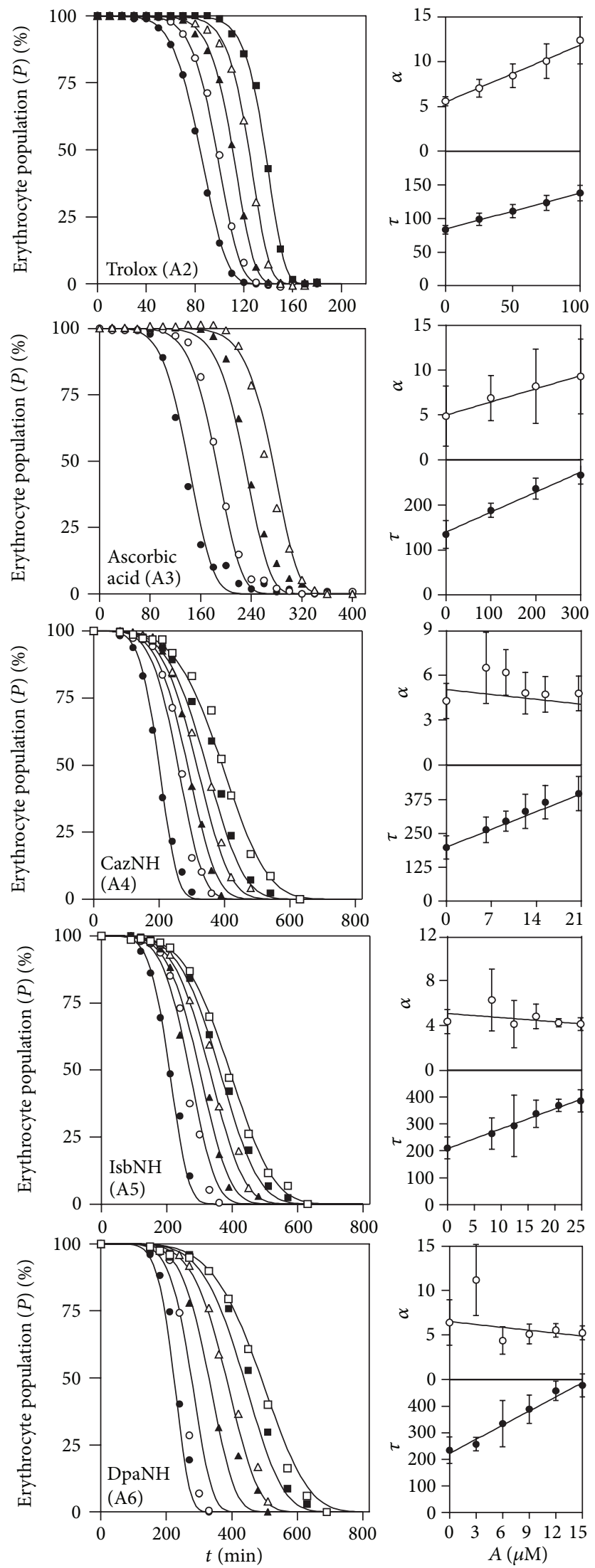

(a) Antioxidant time-dose analysis

Figure 3: Continued. 


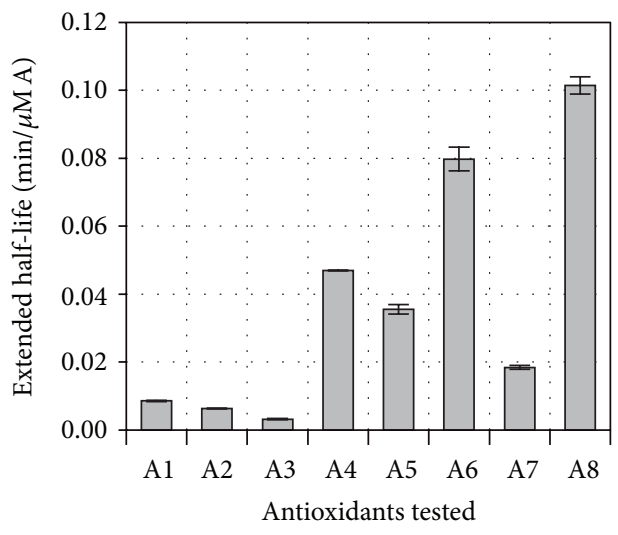

(b) Criteria value for comparison

FIGURE 3: Graphical representation of the time-dose antioxidant responses collected from the bibliography (enumerated in Table 1) adjusted to (7) perturbed by (iv) (Table 3).

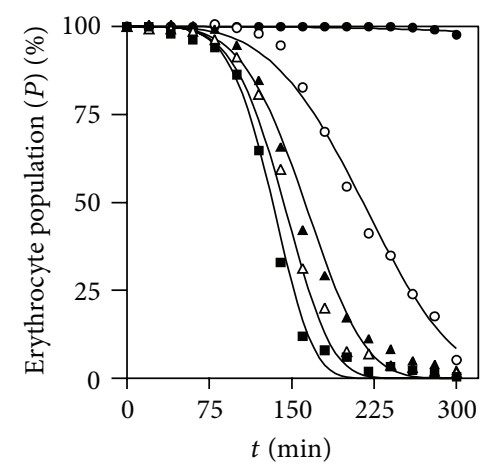

(a) Prooxidant time-dose analysis

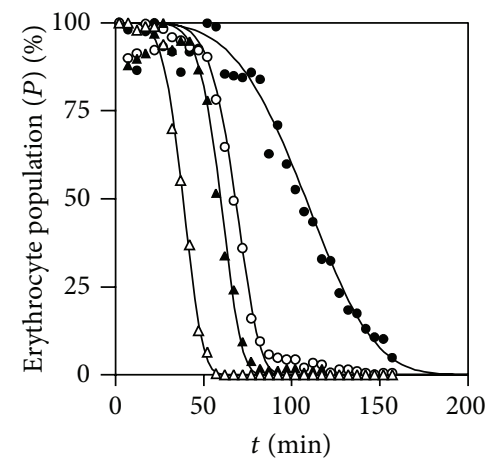

(c) Isotonic resistance test
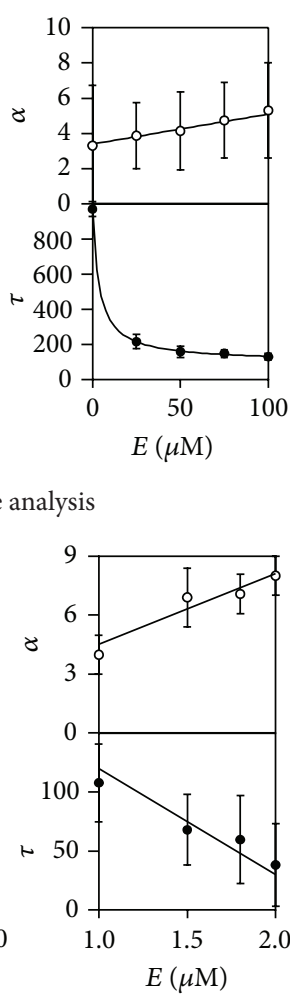

$E(\mu \mathrm{M})$
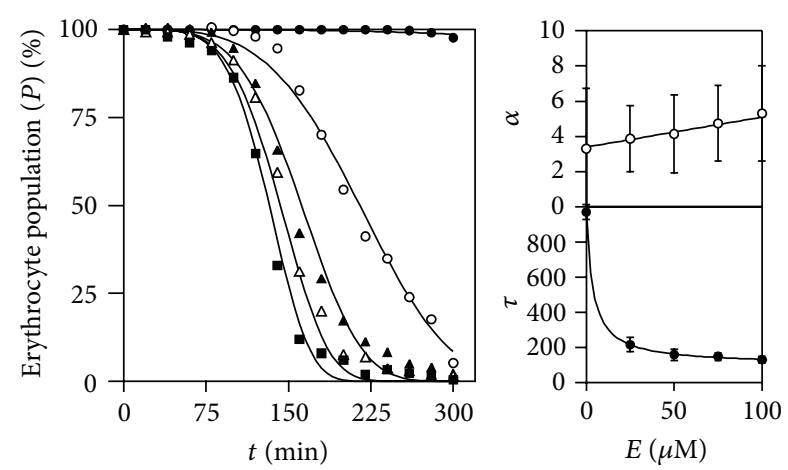

(b) Osmotic fragility test
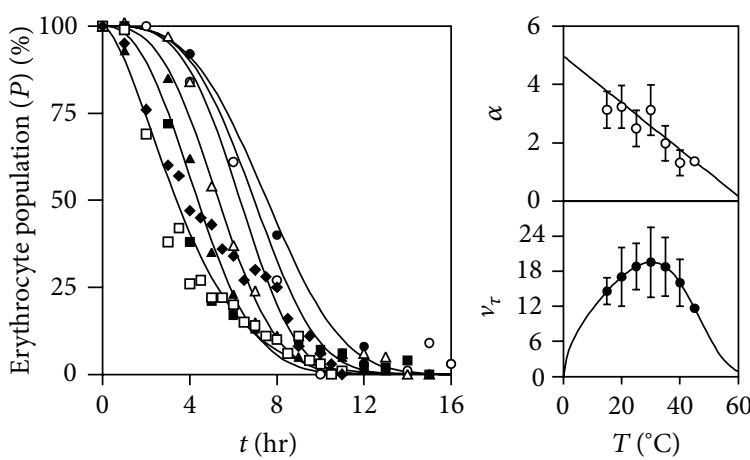

(d) Temperature effect

FIGURE 4: Graphical results of applying the kinetic model (5) or any other reparameterization form to the survival erythrocyte population curve affected by different effectors varying one or more parameters with one or various of the phenomenological functions describedequations ((iv), (v), (vi), and (vii); Table 3).

the specific behavior of the characterizing parameters of (5) and modified by the actions of effectors. The time and effector dependent functions are used, and this produces consistent and meaningful criteria to characterize and quantify the survival erythrocyte population minimizing the effects of the error produced by the experimental conditions.
In this regard, when studying the activity of prooxidant agent AAPH [36] (E1, Table 1) over the kinetic erythrocyte population curve (Figure 4(a)), $H$ decreasing behavior affecting the parameter $\tau$ and $L$ increasing relation for the parameter $\alpha$ are found. Those phenomenological effector responses over the erythrocyte population curve traduce a 
saturated effect produced by the AAPH agent. Figure 4(b) shows a typical response in the osmotic fragility test caused by the surfactant DATB [38] (E2, Table 1). The parametric modifications over the parameters are inversely related to those found for the previous case ( $H$ decreasing relation for $\alpha$ and $L$ increasing one for $\tau$ ). When observing the isotonic resistance test responses [39] (E3, Table 1), using increasing concentrations of ammonium chloride (Figure 4(c)), $L$ modifier relations are found for the parameters $\tau$ and $\alpha$. Finally, using the effect of temperature over kinetic erythrocyte population curve [28], Figure 4(d) shows the case in which a bell type curve is found useful to characterize the effector pattern. For example, we have used the reparametrized form (9), making explicitly the parameter that accounts for the rate of the process when the $50 \%$ of the population are lysed $\left(v_{\tau}, \%\right.$ erythrocyte lysis/hr). The value $v_{\tau}$ is modified by B type function and the $\alpha$ by $\mathrm{L}$ decreasing one. For all the assayed effectors, statistically significant descriptions, with very accurate predictions, were provided.

\section{Conclusions}

We have established that the kinetic model (5) (or any of its other reparameterization forms) describes adequately the variation of the degree of hemolysis as a function of the time. Afterwards a simple bivariate analysis taking into account the time and the antioxidant dose was successfully modeled by means of a simultaneous analysis. Overall, in this paper, a quantification method was developed for the OxHLIA and verified by analyzing the results of other authors that had investigated the capacity of several antioxidants. This analysis reveals the high efficiency of the proposed approach. The model parameters obtained were used to compare the capacity, identify trends, and analyze the dose-equivalent system response, providing more complete information about antioxidant behavior. Therefore, a more efficient way to determine total antioxidant capacities than the simple approach to study the dose-response in a two steps procedure is presented.

In addition, bivariate analysis taking into account the time and other effectors was successfully modeled in similar principles more than those used for antioxidant responses. Using a bivariate model seems to be a disadvantage regarding other apparent simpler routines. In agreement with the opinion of Niki et al. [24], we believe that bivariate approaches of the type proposed here could improve any bioassay in which the inhibitory or stimulatory action of an effector is superposed onto the variation with time of the target system, or the particular time-course of the response is a relevant aspect of that action. Most bioassays that are based on hemolytic processes could be examples on this matter, as it has been demonstrated and discussed above in detail.

\section{Conflict of Interests}

The authors declare that there is no conflict of interests regarding the publication of this paper.

\section{Acknowledgments}

The authors wish to thank Ministerio de Ciencia e Innovación (project CTM2010-18411, co-financed with FEDER funds by European Union) for financial support. Miguel Ángel Prieto Lage was awarded one grant from the JAE predoctoral program cofinanced by the CSIC and European Social Fund (ESF). The authors are also indebted to Isabel Manzano from CZ Veterinaria, S.A., who supplied them with the sheep blood for hemolytic assays performed to contrast the results here presented. Last but not least, the authors want to express their gratitude to Araceli Menduiña Santomé for their professional work.

\section{References}

[1] M. R. McCall and B. Frei, "Can antioxidant vitamins materially reduce oxidative damage in humans?" Free Radical Biology and Medicine, vol. 26, no. 7-8, pp. 1034-1053, 1999.

[2] L. J. Marnett, “Oxyradicals and DNA damage," Carcinogenesis, vol. 21, no. 3, pp. 361-370, 2000.

[3] S. P. Hussain, L. J. Hofseth, and C. C. Harris, "Radical causes of cancer," Nature Reviews Cancer, vol. 3, no. 4, pp. 276-285, 2003.

[4] A. Rahal, A. Kumar, V. Singh et al., "Oxidative stress, prooxidants, and antioxidants: the interplay," BioMed Research International, vol. 2014, Article ID 761264, 19 pages, 2014.

[5] J. M. C. Gutteridge and B. Halliwell, "Antioxidants: molecules, medicines, and myths," Biochemical and Biophysical Research Communications, vol. 393, no. 4, pp. 561-564, 2010.

[6] S. C. Forester and J. D. Lambert, "The role of antioxidant versus pro-oxidant effects of green tea polyphenols in cancer prevention," Molecular Nutrition and Food Research, vol. 55, no. 6, pp. 844-854, 2011.

[7] M. Valko, C. J. Rhodes, J. Moncol, M. Izakovic, and M. Mazur, "Free radicals, metals and antioxidants in oxidative stressinduced cancer," Chemico-Biological Interactions, vol. 160, no. 1, pp. 1-40, 2006.

[8] S. Chatterjee, T. B. Poduval, J. C. Tilak, and T. P. A. Devasagayam, "A modified, economic, sensitive method for measuring total antioxidant capacities of human plasma and natural compounds using Indian saffron (Crocus sativus)," Clinica Chimica Acta, vol. 352, no. 1-2, pp. 155-163, 2005.

[9] N. Pellegrini, M. Serafini, S. Salvatore, D. Del Rio, M. Bianchi, and F. Brighenti, "Total antioxidant capacity of spices, dried fruits, nuts, pulses, cereals and sweets consumed in Italy assessed by three different in vitro assays," Molecular Nutrition and Food Research, vol. 50, no. 11, pp. 1030-1038, 2006.

[10] M. B. Oliveira, A. H. do Prado, J. Bernegossi et al., “Topical application of retinyl palmitate-loaded nanotechnology-based drug delivery systems for the treatment of skin aging," BioMed Research International, vol. 2014, Article ID 632570, 7 pages, 2014.

[11] Y. M. A. Naguib, "A fluorometric method for measurement of oxygen radical-scavenging activity of water-soluble antioxidants," Analytical Biochemistry, vol. 284, no. 1, pp. 93-98, 2000.

[12] E. Niki, "Assessment of antioxidant capacity in vitro and in vivo," Free Radical Biology and Medicine, vol. 49, no. 4, pp. 503-515, 2010.

[13] E. N. Frankel and A. S. Meyer, "The problems of using onedimensional methods to evaluate multifunctional food and 
biological antioxidants," Journal of the Science of Food and Agriculture, vol. 80, pp. 1925-1941, 2000.

[14] E. N. Frankel and J. W. Finley, "How to standardize the multiplicity of methods to evaluate natural antioxidants," Journal of Agricultural and Food Chemistry, vol. 56, no. 13, pp. 4901-4908, 2008.

[15] R. L. Prior and G. Cao, "In vivo total antioxidant capacity: comparison of different analytical methods," Free Radical Biology and Medicine, vol. 27, no. 11-12, pp. 1173-1181, 1999.

[16] M. Carocho and I. C. F. R. Ferreira, "A review on antioxidants, prooxidants and related controversy: natural and synthetic compounds, screening and analysis methodologies and future perspectives," Food and Chemical Toxicology, vol. 51, no. 1, pp. 15-25, 2013.

[17] R. Apak, K. Güçlü, B. Demirata et al., "Comparative evaluation of various total antioxidant capacity assays applied to phenolic compounds with the CUPRAC assay," Molecules, vol. 12, pp. 1496-1547, 2007.

[18] E. N. Frankel, "In search of better methods to evaluate natural antioxidants and oxidative stability in food lipids," Trends in Food Science and Technology, vol. 4, no. 7, pp. 220-225, 1993.

[19] E. N. Frankel, "Methods of evaluating food antioxidants: reply," Trends in Food Science \& Technology, vol. 51, pp. 56-57, 1994.

[20] D. Huang, B. Ou, and R. L. Prior, "The chemistry behind antioxidant capacity assays," Journal of Agricultural and Food Chemistry, vol. 53, no. 6, pp. 1841-1856, 2005.

[21] I. I. Koleva, T. A. van Beek, J. P. H. Linssen, A. de Groot, and L. N. Evstatieva, "Screening of plant extracts for antioxidant activity: a comparative study on three testing methods," Phytochemical Analysis, vol. 13, no. 1, pp. 8-17, 2002.

[22] M. Laguerre, J. Lecomte, and P. Villeneuve, "Evaluation of the ability of antioxidants to counteract lipid oxidation: existing methods, new trends and challenges," Progress in Lipid Research, vol. 46, no. 5, pp. 244-282, 2007.

[23] V. Roginsky and E. A. Lissi, "Review of methods to determine chain-breaking antioxidant activity in food," Food Chemistry, vol. 92, no. 2, pp. 235-254, 2005.

[24] E. Niki, E. Komuro, M. Takahashi, S. Urano, E. Ito, and K. Terao, "Oxidative hemolysis of erythrocytes and its inhibition by free radical scavengers," The Journal of Biological Chemistry, vol. 263, no. 36, pp. 19809-19814, 1988.

[25] D. Bonarska-Kujawa, S. Cyboran, R. Żyłka, J. Oszmiański, and H. Kleszczyńska, "Biological activity of blackcurrant extracts (Ribes nigrum L.) in relation to erythrocyte membranes," BioMed Research International, vol. 2014, Article ID 783059, 13 pages, 2014.

[26] J. Takebayashi, J. Chen, and A. Tai, "A method for evaluation of antioxidant activity based on inhibition of free radical-induced erythrocyte hemolysis," Advanced Protocols in Oxidative Stress II, vol. 594, pp. 287-296, 2010.

[27] E. González, F. Vaillant, G. Rojas, and A. Pérez, "Novel semiautomated method for assessing in vitro cellular antioxidant activity using the light-scattering properties of human erythrocytes," Journal of the Science of Food and Agriculture, vol. 58, pp. 14551461, 2010.

[28] P. Riobó, B. Paz, J. M. Franco, J. A. Vázquez, and M. A. Murado, "Proposal for a simple and sensitive haemolytic assay for palytoxin. Toxicological dynamics, kinetics, ouabain inhibition and thermal stability," Harmful Algae, vol. 7, no. 4, pp. 415-429, 2008 .
[29] A. Banerjee, A. Kunwar, B. Mishra, and K. I. Priyadarsini, "Concentration dependent antioxidant/pro-oxidant activity of curcumin Studies from AAPH induced hemolysis of RBCs," Chemico-Biological Interactions, vol. 174, no. 2, pp. 134-139, 2008.

[30] J. Takebayashi, N. Iwahashi, Y. Ishimi, and A. Tai, “Development of a simple 96-well plate method for evaluation of antioxidant activity based on the oxidative haemolysis inhibition assay (OxHLIA)," Food Chemistry, vol. 134, no. 1, pp. 606-610, 2012.

[31] M. A. Murado and M. A. Prieto, "NOEC and LOEC as merely concessive expedients: two unambiguous alternatives and some criteria to maximize the efficiency of dose-response experimental designs," Science of the Total Environment, vol. 461-462, pp. 576-586, 2013.

[32] J. Takebayashi, H. Kaji, K. Ichiyama et al., "Inhibition of free radical-induced erythrocyte hemolysis by 2-O-substituted ascorbic acid derivatives," Free Radical Biology and Medicine, vol. 43, no. 8, pp. 1156-1164, 2007.

[33] S. Prikler, "Robert de Levie: advanced excel for scientific data analysis, 2nd ed," Analytical and Bioanalytical Chemistry, vol. 395, no. 7, pp. 1945-1946, 2009.

[34] C. Comuzzi, P. Polese, A. Melchior, R. Portanova, and M. Tolazzi, "SOLVERSTAT: a new utility for multipurpose analysis. An application to the investigation of dioxygenated $\mathrm{Co}$ (II) complex formation in dimethylsulfoxide solution," Talanta, vol. 59, no. 1, pp. 67-80, 2003.

[35] M. A. Prieto, J. A. Vázquez, and M. A. Murado, "Hydrolysis optimization of mannan, curdlan and cell walls from Endomyces fibuliger grown in mussel processing wastewaters," Process Biochemistry, vol. 46, no. 8, pp. 1579-1588, 2011.

[36] E. Niki, "Antioxidants in relation to lipid peroxidation," Chemistry and Physics of Lipids, vol. 44, no. 2-4, pp. 227-253, 1987.

[37] Y.-Z. Tang and Z.-Q. Liu, "Free-radical-scavenging effect of carbazole derivatives on AAPH-induced hemolysis of human erythrocytes," Bioorganic and Medicinal Chemistry, vol. 15, no. 5, pp. 1903-1913, 2007.

[38] S. Shalel, S. Streichman, and A. Marmur, "Modeling surfactantinduced hemolysis by Weibull survival analysis," Colloids and Surfaces B: Biointerfaces, vol. 27, no. 2-3, pp. 223-229, 2003.

[39] A. V. Chernyshev, P. A. Tarasov, K. A. Semianov, V. M. Nekrasov, A. G. Hoekstra, and V. P. Maltsev, "Erythrocyte lysis in isotonic solution of ammonium chloride: theoretical modeling and experimental verification," Journal of Theoretical Biology, vol. 251, no. 1, pp. 93-107, 2008.

[40] R. H. Orcutt, T. S. Thurmond, and K. E. Ferslew, "Mathematical modeling of the osmotic fragility of rabbit red blood cells," Journal of Pharmacological and Toxicological Methods, vol. 34, no. 3, pp. 169-174, 1995.

[41] M. A. Murado and J. A. Vázquez, "Mathematical model for the characterization and objective comparison of antioxidant activities," Journal of Agricultural and Food Chemistry, vol. 58, no. 3, pp. 1622-1629, 2010.

[42] P. Terpinc and H. Abramovič, "A kinetic approach for evaluation of the antioxidant activity of selected phenolic acids," Food Chemistry, vol. 121, no. 2, pp. 366-371, 2010.

[43] S. Özilgen and M. Özilgen, "Kinetic model of lipid oxidation in foods," Journal of Food Science, vol. 55, no. 2, pp. 498-501, 1990.

[44] A. DeLean, P. J. Munson, and D. Rodbard, "Simultaneous analysis of families of sigmoidal curves: application to bioassay, radioligand assay, and physiological dose-response curves," The American Journal of Physiology, vol. 235, no. 2, pp. E97-102, 1978. 
[45] J. K. Piotrowski and J. M. Buchanan, "Dose-time-response functions for toxic chemicals," Environmental Monitoring and Assessment, vol. 2, no. 1-2, pp. 139-156, 1982.

[46] M. A. Murado, M. P. González, and J. A. Vázquez, "Doseresponse relationships: an overview, a generative model and its application to the verification of descriptive models," Enzyme and Microbial Technology, vol. 31, no. 4, pp. 439-455, 2002.

[47] S. Goutelle, M. Maurin, F. Rougier et al., "The Hill equation: a review of its capabilities in pharmacological modelling," Fundamental and Clinical Pharmacology, vol. 22, no. 6, pp. 633648, 2008.

[48] J. O. Ragnarsson and T. P. Labuza, "Accelerated shelf-life testing for oxidative rancidity in foods-a review," Food Chemistry, vol. 2, no. 4, pp. 291-308, 1977.

[49] W. Weibull and S. Sweden, "A statistical distribution function of wide applicability," Journal of Applied Mechanics, vol. 18, pp. 293-297, 1951.

[50] Z. H. Kuang, P. F. Wang, R. L. Zheng, Z. L. Liu, and Y. Liu, "Making vitamin C lipo-soluble enhances its protective effect against radical induced hemolysis of erythrocytes," Chemistry and Physics of Lipids, vol. 71, no. 1, pp. 95-97, 1994.

[51] M. A. Murado and M. A. Prieto, "Oversimplification and overstandardization in biological methods: sperm bioassays in ecotoxicology as a case of study and a proposal for their reformulation," The Scientific World Journal, vol. 2014, Article ID 936202, 13 pages, 2014.

[52] S. P. Gieseg and H. Esterbauer, "Low density lipoprotein is saturable by pro-oxidant copper," FEBS Letters, vol. 343, no. 3, pp. 188-194, 1994.

[53] M. A. Prieto, I. Rodríguez-Amado, J. A. Vázquez, and M. A. Murado, " $\beta$-Carotene assay revisited. Application to characterize and quantify antioxidant and prooxidant activities in a microplate," Journal of Agricultural and Food Chemistry, vol. 60, no. 36, pp. 8983-8993, 2012.

[54] M. Á. P. Lage, M. A. M. García, J. A. V. Álvarez, Y. Anders, and T. P. Curran, "A new microplate procedure for simultaneous assessment of lipophilic and hydrophilic antioxidants and prooxidants, using crocin and $\beta$-carotene bleaching methods in a single combined assay: tea extracts as a case study," Food Research International, vol. 53, no. 2, pp. 836-846, 2013. 

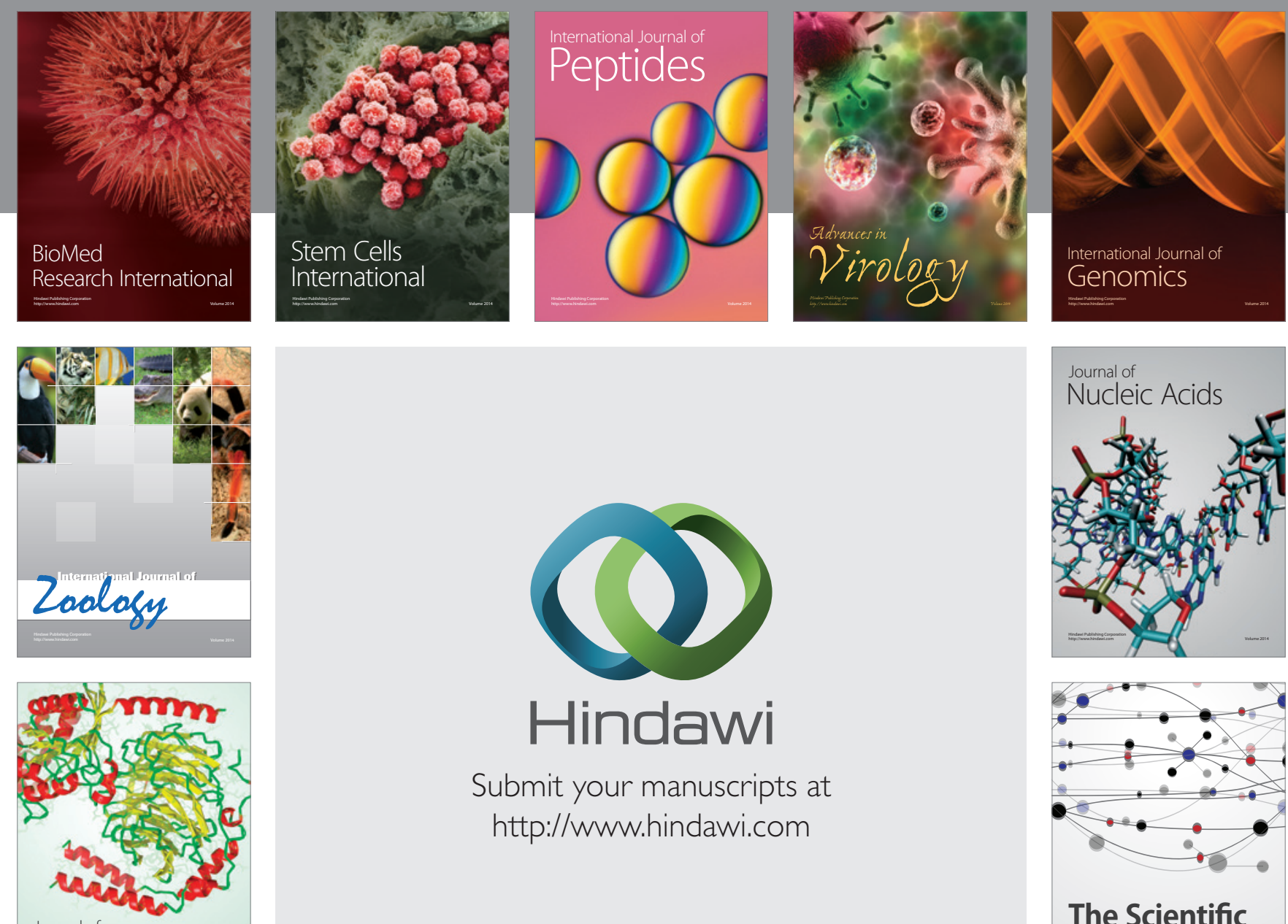

Submit your manuscripts at

http://www.hindawi.com

Journal of
Signal Transduction
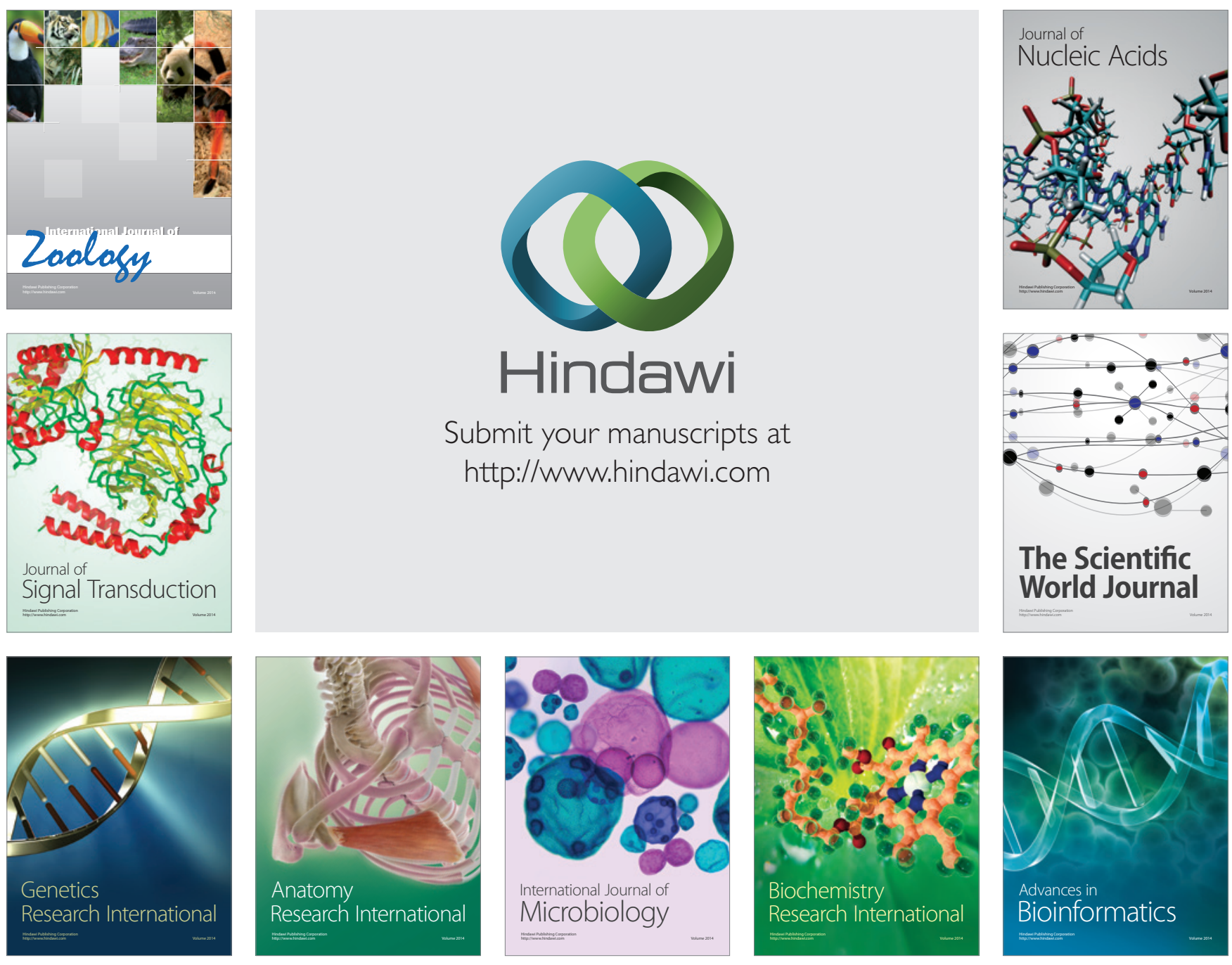

The Scientific World Journal
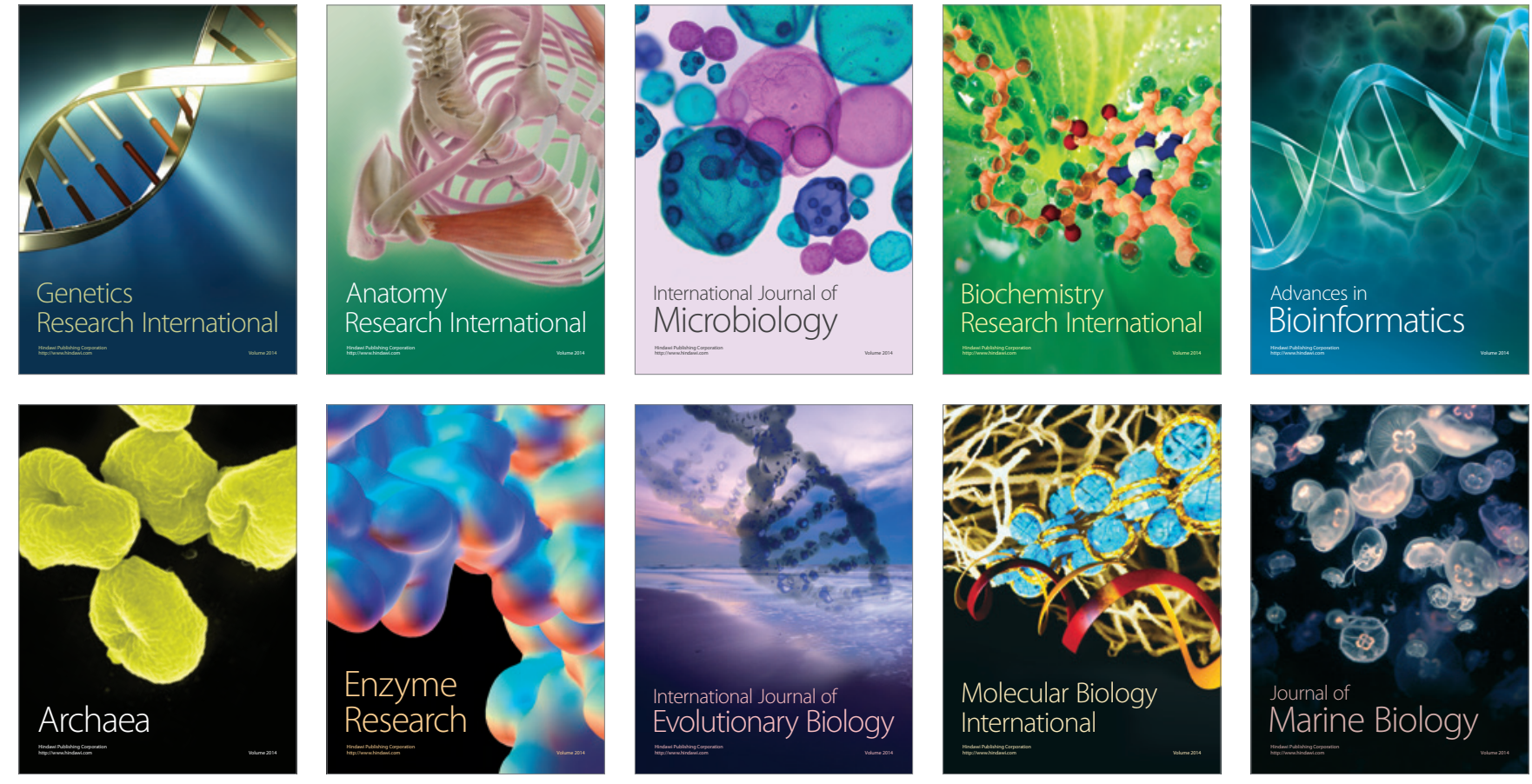